\title{
Paravertebral Extramedullary Tumors with Chronic Myelogenous Leukemia
}

\author{
Tomohiro YAMAKAWA ${ }^{1}$, Mitsutoshi KUROSAWA ${ }^{1}$, Hiroaki SUZUKI ${ }^{2}$ \\ ${ }^{1}$ National Hospital Organization Hokkaido Cancer Center, Department of Hematology \\ ${ }^{2}$ National Hospital Organization Hokkaido Cancer Center, Department of Pathology, Hokkaido, JAPON
}

To Editor,

An 87-year-old man presented with leukocytosis and thrombocytosis. Bone marrow analysis showed markedly myeloid hyperplasia with a slight proliferation of myeloblasts, basophils, and eosinophils. The cytogenetic examination showed Philadelphia chromosome without additional chromosome abnormalities. We diagnosed as chronic myelogenous leukemia (CML) in chronic phase .

Computed tomography (CT) scan of the thorax showed bilateral paravertebral tumors (image1). We performed needle biopsy of the right-sided tumor. The specimen revealed the same pattern as those of bone marrow in microscopic findings (image2 H\&E x 400), flow cytometry analysis, and chromosome analysis. We thought the tumors as extramedullary hematopoiesis.

We started to administer nilotinib $600 \mathrm{mg}$, but we could not help reducing it to $300 \mathrm{mg}$ because of grade 3 thrombocytopenia. He achieved a complete cytogenetic response $(\mathrm{CCyR})$ three months later and paravertebral tumors reduced in CT scan (image 3).

The case of CML with extramedullary blastic infiltrates should be diagnosed and treated as CML in blast crisis. ${ }^{1}$ However, a few cases were reported that extramedullary tumors were the hematopoie-

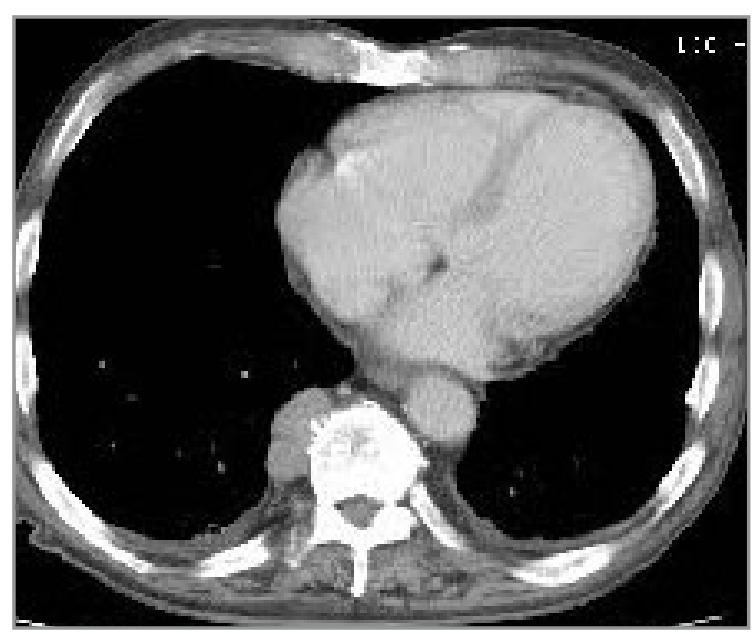

Figure 1. Computed tomography image showing bilateral paravertebral solid masses

sis. ${ }^{2-4}$ Such cases were treated by tyrosine kinase inhibitor and had better clinical courses than those of CML in blast crisis. ${ }^{2-4}$ In our case, low dose nilotinib was effective in treating both extramedullary hematopoiesis and bone marrow. 


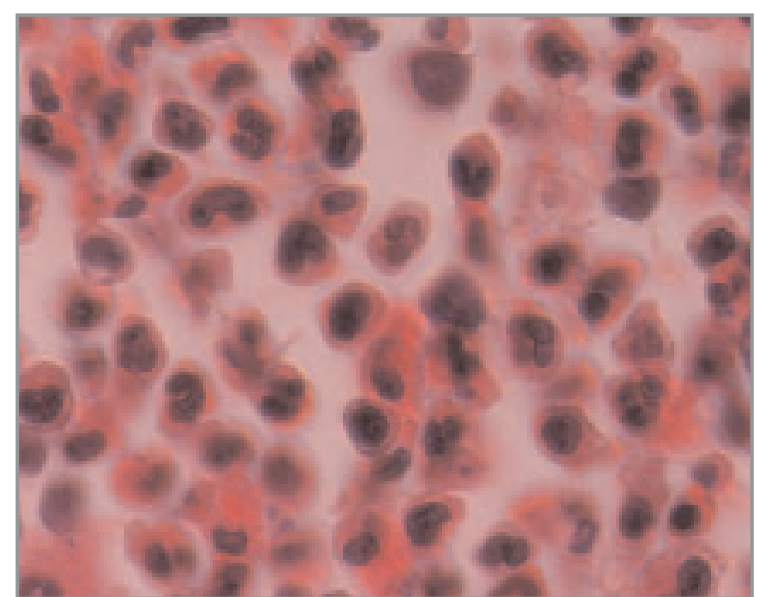

Figure 2. Histopathological examination of paravertebral mass showing marked myeloid hyperplasia with slight proliferation of myeloblasts, basophils and eosinophils ( $\mathrm{H} \& \mathrm{E} x$ 400)

\section{REFERENCES}

1. Baccarani M, Cortes J, Pane F, et al. Chronic myeloid leukemia: an update of concepts and management recommendations of European LeukemiaNet. J Clin Oncol 27: 6041-6051, 2009.

2. Kobayashi Y, Tanaka T, Kawata E, Akaogi T. Chronic myelogenous leukemia in the chronic phase with lymph node swelling which represented extramedullary involvement composed of cells at different stages of maturation. Rinsho Byori 59: 360-363, 2011.

3. Aleem A, Siddiqui N. Chronic myeloid leukemia presenting with extramedullary disease as massive ascites responding to imatinib mesylate. Leuk Lymphoma 46: 1097-1099, 2005.

4. Sakakura M, Ohishi K, Nomura K, et al. Case of chronic-phase chronic myelogenous leukemia with an abdominal hematopoietic tumor of leukemic clone origin. Am J Hematol 77: 167-70, 2004.

\section{Correspondence}

Tomohiro YAMAKAWA

Department of Hematology

National Hospital Organization Hokkido Cancer Center

4-2-3-54, Kikusui, Shiroishi-ku,

Sapporo, Hokkaido

JAPAN

Tel: 011-811-9111

Fax: 011-811-9137

e-mail: tyamakaw@sap-cc.go.jp

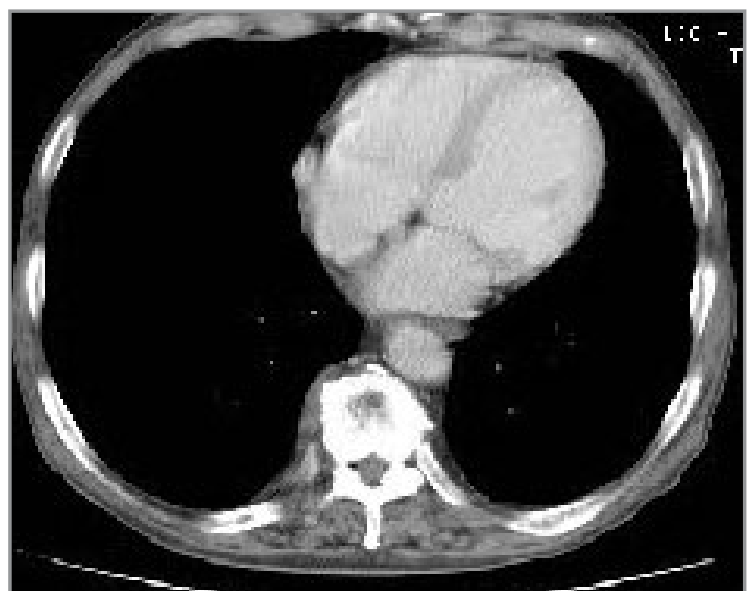

Figure 3. Computed tomography image showing reduced paravertebral masses 